\title{
ASYMPTOTICS OF TWO INTEGRALS FROM OPTIMIZATION THEORY AND GEOMETRIC PROBABILITY
}

\author{
D. J. GATES, ${ }^{*}$ CSIRO Division of Mathematics and Statistics
}

\begin{abstract}
Asymptotic series are derived for two integrals using a Gaussian identity and Laplace's method, demonstrating an improvement over earlier methods.

LAPLACE'S METHOD; OPTIMIZATION
\end{abstract}

Anderssen et al. (1976) obtain various bounds and approximations for the expected distance

$$
m_{k}=\int_{0}^{1} \cdots \int_{0}^{1}\left(x_{1}^{2}+\cdots+x_{k}^{2}\right)^{\frac{1}{2}} d x_{1} \cdots d x_{k}
$$

from the origin of a point uniformly distributed in the cube $[0,1]^{k}$. They evaluate $m_{1}$, $m_{2}$ and $m_{3}$ exactly. Otherwise their computationally most efficient formula, by far, is the asymptotic series

(2) $\quad m_{k}=(k / 3)^{\frac{1}{2}}\left(1-1 / 10 k-13 / 280 k^{2}-101 / 2800 k^{3}-37533 / 1232000 k^{4}\right)+O\left(k^{-\frac{9}{2}}\right)$

as $k \rightarrow \infty$. Terms up to $k^{-3}$ give, for example, $m_{4}$ accurate to five figures, $m_{10}$ accurate to six figures and $m_{20}$ accurate to seven figures. Their derivation of (2) is, however, cumbersome. We give a simple derivation based on Laplace's method.

The authors also study the expected interpoint distances

$$
\left.M_{k}=\int_{0}^{1} \cdots \int_{0}^{1}\left\{x_{1}-y_{1}\right)^{2}+\cdots+\left(x_{k}-y_{k}\right)^{2}\right\}^{\frac{1}{2}} d x_{1} d y_{1} \cdots d x_{k} d y_{k}
$$

but do not give an asymptotic series like (2), presumably because of the work required using their method. We give a simple derivation of such a series, again using Laplace's method.

Since

$$
\lambda^{\frac{1}{2}}=(2 / \pi)^{\frac{1}{2}} \lambda \int_{0}^{\infty} d s \exp \left(-\frac{1}{2} \lambda s^{2}\right)
$$

we can write

$$
m_{k}=(2 / \pi)^{\frac{1}{2}} k \int_{0}^{\infty} f^{\prime}\left(-\frac{1}{2} s^{2}\right) f\left(-\frac{1}{2} s^{2}\right)^{k-1} d s
$$

Received 28 August 1985.

* Postal address: CSIRO Division of Mathematics and Statistics, GPO Box 1965, Canberra ACT 2601, Australia. 
where

$$
f(t)=\int_{0}^{1} \exp \left(t x^{2}\right) d x
$$

Since $f(t)$ has a maximum at $t=0$, and $f^{\prime}(0)=\frac{1}{3}$, we write

$$
\begin{aligned}
f(t) & =\exp (t / 3) \int_{0}^{1}\left\{1+t\left(x^{2}-\frac{1}{3}\right)+\frac{1}{2} t^{2}\left(x^{2}-\frac{1}{3}\right)^{2}+\cdots\right\} d x \\
& =\exp (t / 3)\left(1+2 t^{2} / 45+\cdots\right) .
\end{aligned}
$$

Similarly

$$
f^{\prime}(t)=\exp (t / 3)\left(\frac{1}{3}+4 t / 45+\cdots\right)
$$

Finally

$$
\begin{aligned}
m_{k} & =(2 / \pi)^{\frac{1}{2}} k \int_{0}^{\infty} \exp \left(-k s^{2} / 6\right)\left(\frac{1}{3}-\frac{2}{45} s^{2}+\frac{1}{90} k s^{4}+\cdots\right) d s \\
& =\left(\frac{k}{3}\right)^{\frac{1}{2}}(1-1 / 10 k+\cdots) .
\end{aligned}
$$

Turning now to (3) we have, similarly,

$$
M_{k}=(2 / \pi)^{\frac{1}{2}} k \int_{0}^{\infty} g^{\prime}\left(-\frac{1}{2} s^{2}\right) g\left(-\frac{1}{2} s^{2}\right)^{k-1} d s
$$

where

$$
g(t)=\int_{0}^{1} \int_{0}^{1} \exp \left(t(x-y)^{2}\right) d x d y,
$$

which has a maximum at $t=0$, where $\mathrm{g}^{\prime}=\frac{1}{6}$. Thus we write

$$
g(t)=\exp (t / 6) \sum_{n=0}^{\infty} t^{n} I_{n}
$$

where

$$
I_{n}=\frac{1}{n !} \int_{0}^{1} \int_{0}^{1}\left\{(x-y)^{2}-\frac{1}{6}\right\}^{n} d x d y
$$

and

$$
g^{\prime}(t)=\exp (t / 6) \sum_{n=0}^{\infty} t^{n} J_{n}
$$

where

$$
J_{n}=I_{n} / 6+(n+1) I_{n+1} .
$$

Then $I_{0}=1, I_{1}=0, I_{2}=7 / 360, I_{3}=11 / 5670, J_{0}=1 / 6, J_{1}=7 / 180$ and $J_{2}=137 / 15120$.

Now putting (12) and (13) in (10) and using standard formulae for moments of a normal density gives

$$
M_{k}=(k / 6)^{\frac{1}{2}}\left(1-7 / 40 k-65 / 896 k^{2}+\cdots\right) .
$$

Anderssen et al. (1976) compute $M_{1}, M_{2}$ exactly and $M_{3}, \cdots, M_{10}$ by a slowly 
convergent series method. They also obtain an upper bound

$$
M_{k} \leqq(k / 6)^{\frac{1}{2}}\left[\left\{1+2(1-3 / 5 k)^{\frac{1}{2}}\right\} / 3\right]^{\frac{1}{2}} \text {. }
$$

The table lists the $M_{1}, \cdots, M_{10}$ from Anderssen et al. and their deviations from (14) (as shown) and (15) denoted (14) $-\boldsymbol{M}_{k}$ and (15) $-\boldsymbol{M}_{k}$ respectively. This illustrates the accuracy of (14), for $k$ not too small, while its efficiency is obvious.

\begin{tabular}{rclc}
\hline$k$ & $M_{k}$ & $(14)-M_{k}$ & $(15)-M_{k}$ \\
\hline 1 & 0.33333 & -0.026 & 0.021 \\
2 & 0.52141 & -0.005 & 0.024 \\
3 & 0.66167 & -0.001 & 0.020 \\
4 & 0.77766 & -0.0006 & 0.017 \\
5 & 0.87853 & -0.0003 & 0.015 \\
6 & 0.96895 & -0.0001 & 0.014 \\
7 & $1 \cdot 05159$ & -0.00007 & 0.013 \\
8 & $1 \cdot 12817$ & -0.00004 & 0.012 \\
9 & $1 \cdot 19985$ & -0.00002 & 0.011 \\
10 & 1.26748 & -0.00001 & 0.010 \\
\hline
\end{tabular}

\section{References}

Anderssen, R. S., Brent, R. P., Daley, D. J. And Moran, P. A. P. (1976) Concerning $\int_{0}^{1} \cdots \int_{0}^{1}\left(x_{1}^{2}+\cdots+x_{k}^{2}\right)^{\frac{1}{2}} d x_{1} \cdots d x_{k}$ and a Taylor series method. SIAM J. Appl. Math. 30, 22-30. 